\title{
Perturbative measurement of magnetoresistance
}

Cite as: Rev. Sci. Instrum. 89, 125115 (2018); https://doi.org/10.1063/1.5047661

Submitted: 09 July 2018 . Accepted: 02 December 2018 . Published Online: 21 December 2018

A. B. Oliveira (D, A. C. C. de Melo, R. B. da Costa (D, N. P. Costa, A. Azevedo (D), and C. Chesman

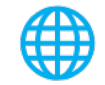

\section{ARTICLES YOU MAY BE INTERESTED IN}

Contributed Review: A review of compact interferometers

Review of Scientific Instruments 89, 121501 (2018); https://doi.org/10.1063/1.5052042

\section{Predicting the failure of pulsed magnets}

Review of Scientific Instruments 89, 124705 (2018); https://doi.org/10.1063/1.5051385

A novel method and system for calibrating the spring constant of atomic force microscope cantilever based on electromagnetic actuation

Review of Scientific Instruments 89, 125119 (2018); https://doi.org/10.1063/1.5051401

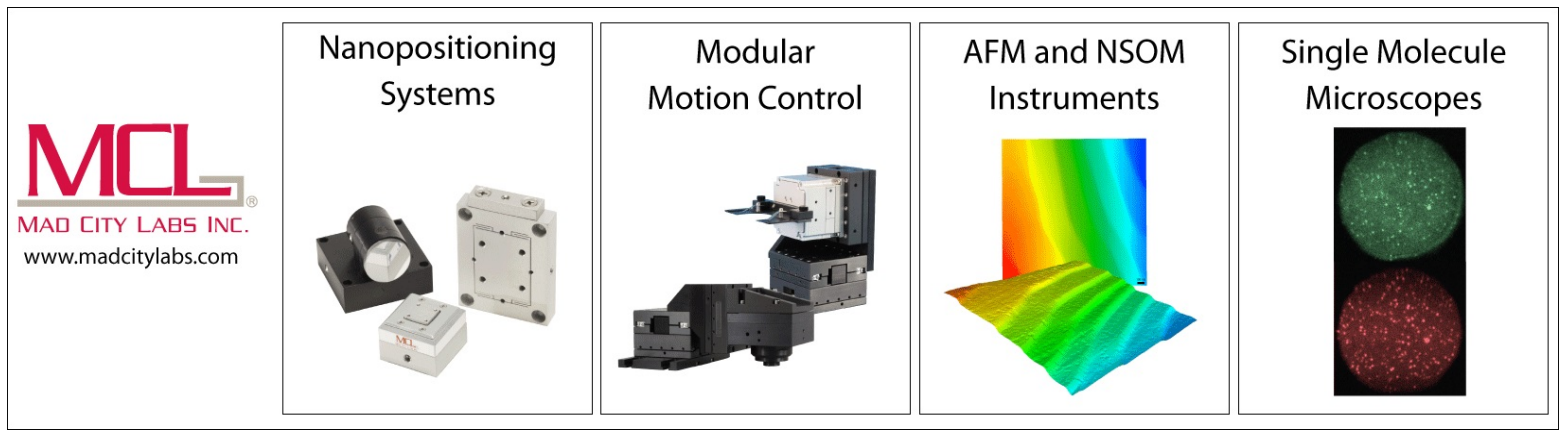




\title{
Perturbative measurement of magnetoresistance
}

\author{
A. B. Oliveira, ${ }^{1,2}$ A. C. C. de Melo, ${ }^{1}$ R. B. da Costa, ${ }^{1}$ N. P. Costa, ${ }^{1}$ \\ A. Azevedo, ${ }^{3}$ and C. Chesman ${ }^{1}$ \\ ${ }^{1}$ Departamento de Física Teórica e Experimental, Universidade Federal do Rio Grande Do Norte, \\ BR-59072-970 Natal, RN, Brazil \\ ${ }^{2}$ Escola de Ciências and Tecnologia Universidade Federal do Rio Grande Do Norte, BR-59072-970 Natal, \\ RN, Brazil \\ ${ }^{3}$ Departamento de Física, Universidade Federal de Pernambuco, 50670-901 Recife, Pernambuco, Brazil
}

(Received 9 July 2018; accepted 2 December 2018; published online 21 December 2018)

\begin{abstract}
In this paper, we report the development of a novel technique in which the magnetoresistance of nanostructures is perturbatively measured by transversally modulating the DC magnetic field. It measures the electrical transport counterpart of the transverse magnetic AC-susceptibility. The technique was developed in a conventional four-probe configuration in which a small DC current flows through the sample and a small transverse AC-field perturbates the equilibrium position of the sample magnetization. Lock-in detection, in-phase with the AC-perturbation, is used to measure the voltage signal between the two inner electrodes of the four-probe station. We successfully demonstrated that this signal is proportional to the product of the first derivative of sample resistance with respect to the equilibrium position of the magnetization times the second derivative of the energy with respect to the equilibrium position of the magnetization. These dependences add new features to the technique investigated here that were not captured by the investigations previously reported on the same subject. To show the effective use of the technique, we discuss its application in measuring magnetic properties of thin magnetic films in the non-saturated regime. Published by AIP Publishing. https://doi.org/10.1063/1.5047661
\end{abstract}

\section{INTRODUCTION}

Measurements of the magnetic properties of nanostructures are usually performed by means of a variety of techniques which depending on the type of the external excitation and the time scale of response can be considered as static, quasi-static, or dynamic techniques. ${ }^{1,2}$ The basic principles on which the experimental techniques are created rely on different magnetic phenomena, such as magnetic induction, ${ }^{3-6}$ magnetic force, ${ }^{7,8}$ magneto-optical properties, ${ }^{9-11}$ magnetic resonances, ${ }^{12-15}$ light scattering by magnetic excitations, ${ }^{16}$ and magnetoresistance. ${ }^{17,18}$ To be suitable for investigation of magnetic properties of nanostructures, the development of new techniques has to consider two key aspects: (i) being able to measure weak signals and (ii) providing information on magnetic phenomena that are not easily obtained by wellestablished techniques. For example, the magnetic field values in which the magnetization reversal processes start in magnetic nanostructures ${ }^{19}$ are not easily measured by existent techniques. Therefore, the development of magnetic instrumentation dealing with weak signals, which operates in the non-saturation of the magnetization regime of field values, is of current interest.

Here we present the development of a perturbative measurement technique based on magnetoresistance effects, termed Perturbative Magnetoresistance (PMR), which can be used to characterize the magnetic properties of nanostructures in magnetic field ranges of non-saturation of the magnetization regime. The physical principle of the PMR can be understood as an extension of the AC-AMR measurements introduced by Venus et $a l .{ }^{20}$ In the AC-AMR technique, the authors used a double modulation detection where the magnetization was perturbed by means of a small AC-field $(100 \mathrm{~Hz})$ superimposed with an AC-current $(40 \mathrm{kHz})$ that flows through the sample. In Ref. 20, the double modulation detection was carried out with no external DC-field. In the PMR technique, the DC-field is always applied to the sample; therefore, the magnetization direction can be slowly reversed in the presence of a perpendicular weak oscillating field. The electrical excitation is obtained by applying a DC current through the sample, and the PMR signal is measured by means of a lock-in amplifier phase-locked to the reference signal. The principle of operation of the perturbative magnetoresistance (PMR) technique and the required instrumentation are described in Sec. II. Section III describes the interpretation of the data with an analytical model. This section also includes the validation of the technique and comparison of the data obtained by means of PMR with data obtained by the conventional techniques of the vibrating sample magnetometer (VSM) and conventional collinear magnetoresistance (MR). Conclusions are presented in Sec. IV.

\section{PERTURBATIVE MAGNETORESISTANCE: PRINCIPLE OF OPERATION AND INSTRUMENTATION}

In the PMR setup, perpendicular AC and DC magnetic fields are simultaneously applied to the sample. As shown below, this configuration makes the measured response be proportional to $d R / d h_{a c}$, where $R$ is the sample resistance and $h_{a c}$ is the perturbative magnetic field. On the other hand, if the AC field is applied parallel to the DC field, the response is proportional to $d R / d H_{d c}{ }^{21}$ While the parallel configuration has been named magnetoresistance AC-susceptibility, 


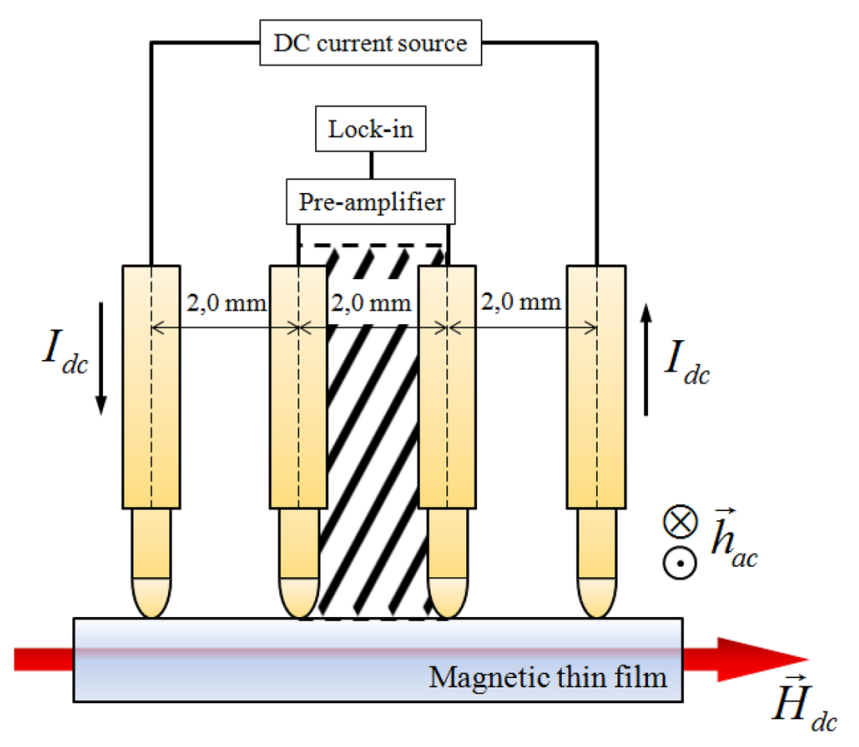

FIG. 1. Illustration of the PMR setup. While the fixed DC-current is applied between the outer probes, the voltage is measured between the inner probes. A spurious signal is induced between the inner probes due to the magnetic flux variation. The lock-in reference signal is provided by the same signal generator used to feed the AC field.

we named the perpendicular configuration as perturbative magnetoresistance.

To avoid any contact-resistance contribution, we employed the collinear four-point probe technique. ${ }^{22}$ Figure 1 illustrates the experimental setup used to perform PMR measurements. Four gold-coated spring contact probes were tightly pressed onto the sample surface, the DC electrical current is applied between the two outer probes, and the voltage signal is measured between the two inner contacts. The current varies from hundreds of microamps to few milliamps. In some measurements, we used an additional low-noise passband preamplifier (Stanford SR560) before the lock-in detection. The DC field is generated by using an electromagnet, and the perpendicular AC field is generated by using a pair of Helmholtz coils which is sinusoidally driven by a $90 \mathrm{~W}$ audio amplifier. In a typical measurement, the amplitude and frequency of the sinusoidal AC field at the sample are 1.0 Oe and $470 \mathrm{~Hz}$, respectively. Since we have time dependent magnetic fields and closed circuits through the probes, two spurious signals need to be eliminated before measurement takes place: (i) An induced AC voltage, due to the perturbative magnetic flux, is generated in between the inner probes by $h_{a c}$ (hatched area of Fig. 1). Before turning on the DC power supply, this spurious AC signal is detected by measuring the 1st harmonic component of the lock-in amplifier. This constant background signal (typically $80 \mu \mathrm{V}$ ) does not depend on the DC field and is later subtracted from the magnetic field dependent signal. (ii) As the DC field is stepped upward or downward, there also appears a spurious contribution to the signal. To eliminate this spurious signal, the data acquisition program has a dead time between the acquisition of two consecutive data points that can be adjusted from $1.0 \mathrm{~s}$ to $15 \mathrm{~s}$.

The physical principle of the PMR technique relies on the change in the electrical resistance of the sample when the

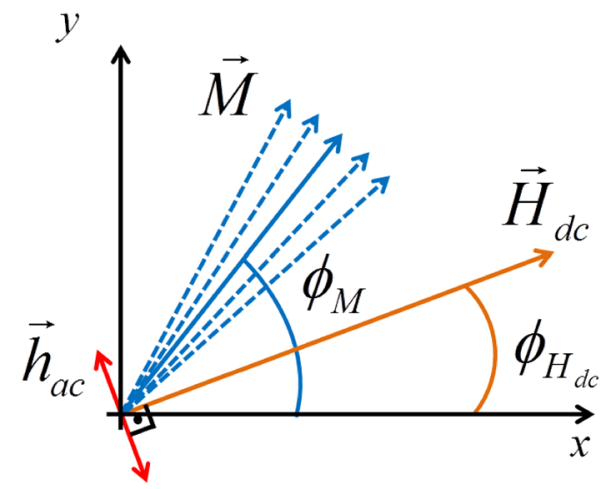

FIG. 2. Illustration of the equilibrium position of the magnetization $\boldsymbol{M}$ in the presence of the external magnetic fields $H_{d c}$ and $h_{a c}$. The dashed arrows represent the time-varying positions of magnetization, oscillating around the equilibrium position given by $\phi_{M}$. Here, the uniaxial anisotropy field is parallel to the $x$-axis.

magnetization weakly oscillates around its equilibrium position under the action of a weak time varying and spatially uniform external magnetic field. Figure 2 shows the vector configuration of the magnetization $(\boldsymbol{M})$ and applied magnetic fields $\boldsymbol{H}_{d c}$ and $\boldsymbol{h}_{a c}$. The in-plane equilibrium position of $\boldsymbol{M}$ is given by the angle $\phi_{M}$, and the time varying positions are illustrated by dashed arrows. Due to $h_{a c}$, the magnetization vector will oscillate around its equilibrium position that is obtained by minimization of the Zeeman and anisotropy energies. ${ }^{23}$ As the electrical resistance weakly oscillates, all the harmonic components can be measured by means of the lock-in amplifier. By varying the magnetic DC-field, the equilibrium position of the magnetization $\phi_{M}\left(H_{d c}, h_{a c}\right)$ and the electrical resistance $R=$ $V\left(H_{d c}, h_{a c}\right) / I_{d c}$ vary as well. Here, $V$ is the measured voltage and $I_{d c}$ is the DC-electrical current, which is kept constant. Thus, for a fixed value of $H_{d c}$ and considering $h_{a c}$ as a perturbation (smaller than the anisotropy fields involved), we can expand $V\left(H_{d c}, h_{a c}\right)$ using Taylor's series expansion as

$$
\begin{aligned}
V\left(H_{d c}, h_{a c}\right)= & V\left(H_{d c}, 0\right)+\left.\frac{\partial V}{\partial h_{a c}}\right|_{h_{a c}=0} h_{a c} \\
& +\left.\frac{1}{2} \frac{\partial^{2} V}{\partial h_{a c}^{2}}\right|_{h_{a c}=0} h_{a c}^{2}+\cdots,
\end{aligned}
$$

where $h_{a c}(t)=h_{0} \cos \omega t, h_{0} \cong 1.0 \mathrm{Oe}$, and $f=\omega / 2 \pi=470 \mathrm{~Hz}$. We used a lock-in amplifier (model EG\&G 7260) to measure the first harmonic component of $V\left(H_{d c}, h_{a c}\right)$, in-phase with the external AC-excitation. As the period of $h_{a c}$ is around $2 \mathrm{~ms}$, the time constant of the lock-in low-pass filter can be as low as $50 \mathrm{~ms}$ with no change in the result. Depending on the level noise, the time constant can be as high as $500 \mathrm{~ms}$.

Defining $V_{P M R}$ as the amplitude of the 1 st harmonic term of Eq. (1), it can be written in terms of different variables,

$$
\begin{aligned}
V_{P M R} & =\left.h_{0} \frac{\partial V}{\partial h_{a c}}\right|_{h_{a c}=0}=\left.h_{0} I_{d c} \frac{\partial R}{\partial h_{a c}}\right|_{h_{a c}=0} \\
& =\left.h_{0} I_{d c} \frac{\partial R}{\partial \phi_{M}} \frac{\partial \phi_{M}}{\partial h_{a c}}\right|_{h_{a c}=0} .
\end{aligned}
$$

To correctly interpret the $V_{P M R}$ signal, we need to know the analytical expressions for both $\partial R / \partial \phi_{M}$ and $\partial \phi_{M} / \partial h_{a c}$ terms that appear in the above equation. The term $\partial R / \partial \phi_{M}$ 
accounts for the modulation of the resistance and depends on the underlying mechanism responsible for the magnetotransport change. The term $\partial \phi_{M} / \partial h_{a c}$ depends on the equilibrium position and is obtained by minimizing the free energy of the sample that contains all the relevant contributions to the magnetic free energy such as Zeeman, anisotropy, demagnetization, and exchange coupling. Therefore, the equilibrium position of magnetization $\left(\phi_{M_{0}}\right)$ is obtained from the minimization conditions of the magnetic free energy $E$ given by

$$
\left.\frac{\partial E}{\partial \phi_{M}}\right|_{\phi_{M_{0}}}=0 \text { and }\left.\frac{\partial^{2} E}{\partial \phi_{M}^{2}}\right|_{\phi_{M_{0}}}>0 .
$$

From the total derivative of $\partial E / \partial \phi_{M}$ with respect to $h_{a c}$, we obtain the expression for $\partial \phi_{M} / \partial h_{a c}$,

$$
\begin{aligned}
\frac{d}{d h_{a c}}\left(\frac{\partial E}{\partial \phi_{M}}\right) & =\left[\frac{\partial}{\partial \phi_{M}}\left(\frac{\partial E}{\partial \phi_{M}}\right)\right] \frac{\partial \phi_{M}}{\partial h_{a c}}+\frac{\partial}{\partial h_{a c}}\left(\frac{\partial E}{\partial \phi_{M}}\right) \\
& =\frac{\partial^{2} E}{\partial \phi_{M}^{2}} \frac{\partial \phi_{M}}{\partial h_{a c}}+\frac{\partial^{2} E}{\partial h_{a c} \partial \phi_{M}}=0 .
\end{aligned}
$$

Therefore the $\partial \phi_{M} / \partial h_{a c}$ term is given by

$$
\frac{\partial \phi_{M}}{\partial h_{a c}}=-\frac{\frac{\partial^{2} E}{\partial h_{a c} \partial \phi_{M}}}{\frac{\partial^{2} E}{\partial \phi_{M}^{2}}}, h_{a c} \rightarrow 0,
$$

where the derivatives are evaluated at the equilibrium position $\phi_{M_{0}}$.

To calculate the $\partial R / \partial \phi_{M}$ term, we consider the anisotropic magnetoresistance (AMR) effect ${ }^{17}$ as the mechanism responsible for the magnetoresistance. Thus,

$$
\frac{\partial R}{\partial \phi_{M}}=-\Delta R \sin (2 \phi)
$$

where $\Delta R=R_{/ /}-R_{\perp}$ is the $\mathrm{AMR}$ amplitude, $R_{\perp}$ is the resistance when DC current is applied perpendicular to $M, R_{/ /}$is the resistance when DC current is applied parallel to $\boldsymbol{M}$, and $\phi$ is the angle between the magnetization and the DC-electrical current direction. As mentioned above, the terms given by Eqs. (5) and (6) are fundamental to interpret the measurement of the PMR signal given by Eq. (2).

\section{THEORETICAL MODEL AND INTERPRETATION OF EXPERIMENTAL DATA}

In this section, we apply the theoretical model developed in Sec. II to investigate the PMR of ferromagnetic thin films. By writing the magnetic free energy of the system and then using the minimization procedure, we obtain the PMR signal as given by Eq. (2). Later, this theoretical model will be applied to explain measurements carried out in polycrystalline thin films of permalloy $\left(\mathrm{Ni}_{80} \mathrm{Fe}_{20}\right)$. The magnetic free energy density includes two contributions, Zeeman and uniaxial anisotropy energies, given by

$$
\varepsilon=-\boldsymbol{M} \cdot\left(\boldsymbol{H}_{d c}+\boldsymbol{h}_{\boldsymbol{a c}}\right)-K_{u}\left(\frac{\boldsymbol{M} \cdot \hat{\boldsymbol{u}}}{M}\right)^{2},
$$

where $\boldsymbol{M}$ is the saturation magnetization, $\boldsymbol{H}_{\boldsymbol{d c}}$ and $\boldsymbol{h}_{\boldsymbol{a c}}$ are the static and time-varying magnetic fields, $\hat{\boldsymbol{u}}$ defines the uniaxial anisotropy direction, $K_{\mathrm{u}}$ is the uniaxial anisotropy constant such that the uniaxial anisotropy field is given by $\boldsymbol{H}_{\boldsymbol{u}}=\left(2 K_{u} / M\right) \hat{\boldsymbol{u}}$. In terms of the angles shown in Fig. 2, where the $x$-axis is parallel to $\hat{\boldsymbol{u}}$, the energy density is given by

$$
\begin{aligned}
\varepsilon= & -M H_{d c} \cos \left(\phi_{M}-\phi_{H_{d c}}\right)-M h_{a c} \cos \left(\phi_{M}-\phi_{h_{a c}}\right) \\
& -\frac{1}{2} M H_{u} \cos ^{2}\left(\phi_{M}\right) .
\end{aligned}
$$

Equation (2) requires the calculation of the second derivative of the energy with respect to $h_{a c}$ and $\phi_{M}$. Thus,

$$
\frac{\partial^{2} \varepsilon}{\partial h_{a c} \partial \phi_{M}}=-M \cos \left(\phi_{M}-\phi_{H_{d c}}\right)
$$

and

$$
\begin{aligned}
\frac{\partial^{2} \varepsilon}{\partial \phi_{M}^{2}}= & M H_{D C} \cos \left(\phi_{M}-\phi_{H_{d c}}\right)+M h_{a c} \sin \left(\phi_{M}-\phi_{H_{d c}}\right) \\
& +M H_{u} \cos \left(2 \phi_{M}\right),
\end{aligned}
$$

where $\phi_{h_{a c}}=\phi_{H_{d c}}+\frac{\pi}{2}$. Using Eqs. (9) and (10) and taking limit of $h_{\mathrm{ac}} \rightarrow 0$, the PMR signal given by Eq. (2) becomes

$$
V_{P M R}=h_{0} I_{d c} \Delta R \frac{\sin \left(2 \phi_{M}\right) \cos \left(\phi_{M}-\phi_{H_{d c}}\right)}{H_{d c} \cos \left(\phi_{M}-\phi_{H_{d c}}\right)+H_{u} \cos \left(2 \phi_{M}\right)},
$$

where the DC-electrical current is applied parallel to the hard axis of magnetization, $y$-axis of Fig. 2. It is important to mention that to interpret the PMR data with Eq. (11). Finally, we need to calculate the equilibrium position of the magnetization as a function of $H_{d c}$, using Eq. (8). Also, in Eq. (6), we used $\phi=\phi_{M}-\phi_{I}=\phi_{M}-\frac{\pi}{2}$.

\section{A. PMR signal validation}

In this subsection, we discuss experimental data that are used to confirm the theoretical interpretation given previously. For instance, Eq. (2) shows that the PMR signal depends linearly on both the intensity of the DC-current $\left(I_{d c}\right)$ and the AC-field amplitude $\left(h_{0}\right)$. This linear dependence is only valid in the limit of $h_{a c} \rightarrow 0$, in which Taylor's expansion is applicable. For $h_{0}$ values higher than a threshold value, the linear dependence given by Eq. (2) is no more valid. It is important to mention that this threshold value depends on intrinsic magnetic parameters of the sample under investigation. To discuss the above assumptions, we investigate a sample with large PMR signal, a bilayer of $\mathrm{Py}(10 \mathrm{~nm}) / \mathrm{CoO}(3 \mathrm{~nm}) / \mathrm{MgO}(100)$. Here, Py is a permalloy $\left(\mathrm{Ni}_{80} \mathrm{Fe}_{20}\right)$ film deposited on a very thin layer of $\mathrm{CoO}$, which is not in the antiferromagnetic phase. The films were sputter deposited on a substrate of $\mathrm{MgO}(100)$ by the $\mathrm{DC}$ magnetron sputtering technique.

Figures 3(a) and 3(b) show the DC field dependence of the PMR signal for different $I_{d c}$ and $h_{0}$ values. Figure 3(a) shows the PMR signal as a function of $H_{\mathrm{dc}}$ for different $h_{0}$ values, where $I_{d c}$ was kept constant at $4.0 \mathrm{~mA}$ and $h_{0}$ was varied from 0 to 9 Oe. The inset shows the PMR dependence for larger values of $H_{\mathrm{dc}}$. The field value, where the PMR signal nulls, is named transition field $\left(H_{t}\right)$. In the perturbative approximation, the $H_{t}$ value should not depend on the external excitation $h_{0}$. As shown in Fig. 3(c), $H_{t}$ remains almost constant for 

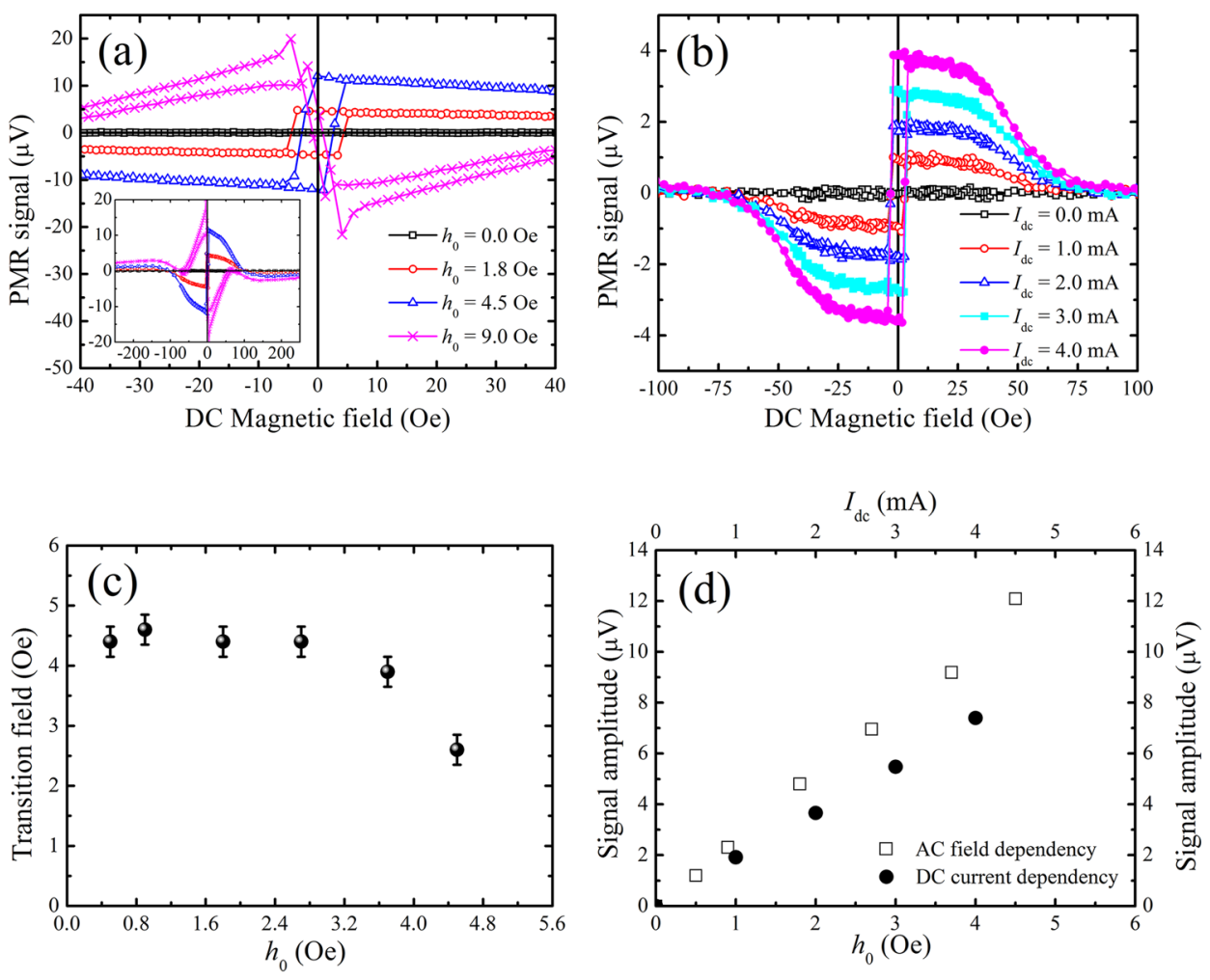

FIG. 3. (a) and (b) show the PMR signal as a function of $H_{\mathrm{dc}}$, measured at room temperature for the sample $\mathrm{Py}(10$ $\mathrm{nm}) / \mathrm{CoO}(3 \mathrm{~nm}) / \mathrm{MgO}(100)$. In (a), the DC current is fixed at $4.0 \mathrm{~mA}$ and the AC field amplitude is varied. In (b), the AC field amplitude is fixed at 2.9 Oe and the DC current is varied. (c) Transition field dependency as a function of $h_{0}$. (d) Linear behavior of the amplitude of the PMR signal as a function of $h_{0}$, for $I_{d c}=4.0 \mathrm{~mA}$ (hollow squares), and as a function of $I_{d c}$ for $h_{0}=2.9 \mathrm{Oe}$ (solid circles). $0<h_{0} \leq 2.5$ Oe, but it decreases for values higher than 2.5 Oe. Therefore, $h_{0}=2.5$ Oe characterizes the upper limit of the AC field and is the maximum field that should be used as the perturbative excitation for this sample. As shown by the pink symbols in Fig. 3(a), the PMR dependence with $H_{\mathrm{dc}}$ changes strongly for $h_{0} \geq 9.0$ Oe. In Fig. 3(b), the AC-field amplitude $\left(h_{0}\right)$ is kept constant at $2.9 \mathrm{Oe}$, while $I_{d c}$ is varied. In this case, the dependence of the PMR signals with the magnetic field is a well behaved and symmetrical hysteresis curve. The $H_{t}$ values do not depend on $I_{d c}$, and the PMR amplitude exhibits linear behavior as a function of both the AC-field amplitude and DC-current, as expected from the model introduced in Sec. III [Eq. (2)]. The value of the signal amplitude, shown in the vertical axis of Fig. 3(d), was obtained by taking the difference between maximum and minimum signals, divided by 2, i.e., sign.amp. $=\frac{P M R_{\max }-P M R_{\min }}{2}$.

\section{B. Experimental results and physical interpretation}

The experimental data discussed in this subsection were obtained by investigating the PMR signal of a single film of permalloy, sputter deposited on $\mathrm{Si}(100)$ with a thickness of $36 \mathrm{~nm}$. To induce a large in-plane uniaxial anisotropy, the film was obliquely deposited with a tilt angle of $75^{\circ}$. As known, obliquely deposited metal films develop columnar microstructures that induce very large uniaxial anisotropy fields as well as large coercivities. ${ }^{24,25}$ Before the PMR measurements, the Py film was investigated by means of magnetoresistance (MR) and vibrating sample magnetometer (VSM) techniques. To understand the physical meaning of the PMR signals, we compared them with the magnetization hysteresis measurements obtained with VSM. Figure 4(a) shows the magnetization curve (black symbols) and PMR signal (blue symbols) as a function of the DC field applied along the easy axis of magnetization. As the Py film was obliquely grown, it exhibits a coercive field of $H_{C} \cong 32 \mathrm{Oe}$ which is larger than typical values of $H_{C}$ for Py polycrystalline films. A PMR signal, of few $\mu \mathrm{V}$, was obtained by applying a DC current of $3.0 \mathrm{~mA}$ and $h_{0} \cong 1.0$ Oe. Observe that in the saturated regime $(H>$ $H_{\text {sat }}$ and $\phi_{M}=\phi_{H_{d c}}=0$ or $\left.\pi\right), V_{\text {PMR }}$ is zero as expected from Eq. (11). Figure 4(b) shows a zoomed-in view of Fig. 4(a) in which the transition field $\left(H_{t} \cong 38 \mathrm{Oe}\right)$ measured by the PMR signal occurs for a field value larger than the coercive field value $\left(H_{C} \cong 32 \mathrm{Oe}\right)$. This is an important property of the transition field measured by the PMR technique. As the DC field value decreases, for instance, along the upper branch of the hysteresis curve, and the magnetization starts to deviate from the saturated state, the PMR signal smoothly increases (blue symbols) reaching a maximum at around the value of the coercive field, and then as the field increases, it suddenly jumps down. The region in which the PMR signal jumps from the maximum to the minimum value occurs in the region between the two vertical dashed lines, which is defined as the switching field distribution (SFD). SFD is the region between the fields at which the magnetization drops to $+90 \%$ to $-90 \%$ of the saturation value. To compare with a quantitative model, we calculated the PMR signal, supposing the simplified model of Stoner-Wohlfarth (SW) described by Eqs. (8)-(11), in which the magnetization direction was obtained by numerical energy minimization, using $H_{u}=55 \mathrm{Oe}$. The PMR signals, given by the solid red and blue lines, exhibit a qualitative agreement with the experimental data showing a sharp peak at the transition field, $H_{t}$. Actually, a model to explain the experimental PMR data needs to take into account the detailed multidomain magnetic configuration as well as the Py grain distribution. Magnetoresistance data exhibit the same behavior as the PMR 



FIG. 4. Measurements performed with DC field applied parallel to the easy axis of magnetization for the obliquely deposited $\mathrm{Py}(36 \mathrm{~nm}) / \mathrm{Si}(100)$. (a) Magnetization curve (black symbols) and PMR signal (blue symbols). (b) Zoomed-in view of (a) showing the down-field sweep (blue symbols) and the up-field sweep (red symbols) of the PMR signal. The solid lines represent the PMR signal calculated using the SW model of coherent rotation of the magnetization. In spite of being an approximation, the sharp peaks are associated with the transition field measured experimentally. (c) MR data in which the down-field sweep is given by the blue solid circles and the up-field sweep by the solid red circles, superimposed to the PMR signal.

signal as shown in Fig. 4(c), where the MR data (solid circles) are superimposed to the PMR signal (open circles).

Figure 5(a) shows the magnetization curve (black symbols) and PMR signal (blue symbols) as a function of the DC field applied along the hard axis of magnetization. The SW scenario, when the magnetic field applied is along the hard axis, predicts that the magnetization equilibrium angle rotates
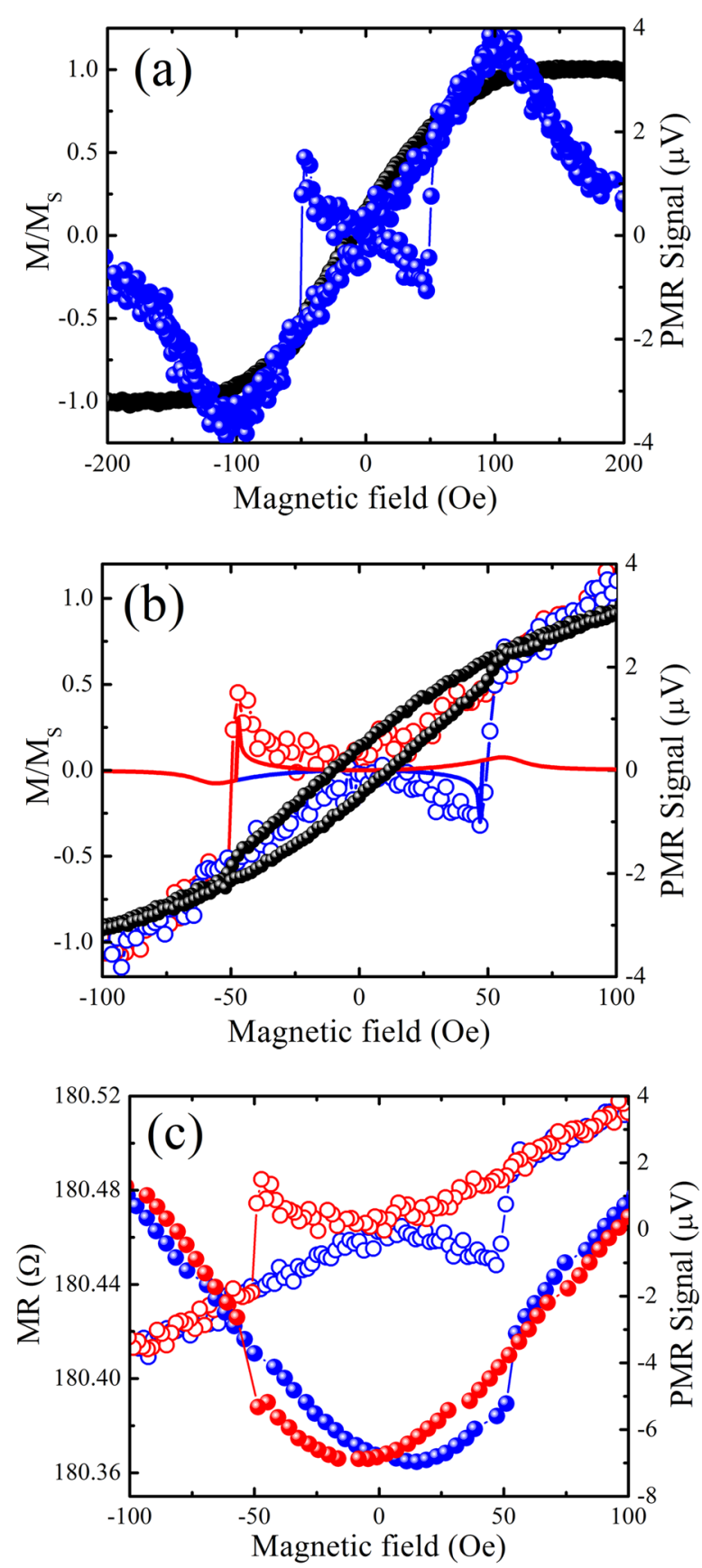

FIG. 5. Measurements performed with the DC magnetic field applied parallel to the hard axis of magnetization for the obliquely deposited Py (36 nm)/Si(100). (a) Magnetization curve (black symbols) and PMR signal (blue symbols). (b) Zoomed-in view of (a) showing the down-field sweep (red symbols) and the up-field sweep (blue symbols) of the PMR signal. The solid lines were numerically obtained from the SW model that considers coherent rotation of the magnetization. The sharp peaks of the PMR signal are associated with the switching field that shows a small variation at the VSM curve. (c) MR data in which the down-field sweep is given by the red solid circles and the up-field sweep by the blue circles, superimposed to the PMR signal.

continuously toward either side of the hysteresis curve with no switching field and no hysteresis. ${ }^{26,27}$ In the sample investigated here, the magnetization curve shown in Fig. 5(a) exhibits a quasi-typical behavior of a hard axis curve with a saturation field value of around $125 \mathrm{Oe}$, a small hysteresis, and a very weak magnetization switching at \pm 50 Oe. The weak magnetization switching, which is hard to be measured by VSM, can 
easily be detected by the PMR technique, as given by the abrupt transitions also at \pm 50 Oe, as shown in Fig. 5(a). Figure 5(b) shows a zoomed-in view of Fig. 5(a), in which the PMR signal is given by the red and blue open circles. The red and blue solid lines were obtained by means of the SW model, similar as in Fig. 4(b), but now considering the DC field applied parallel to the hard axis of magnetization. This is a clear demonstration that PMR can be used to detect feeble variation of the magnetization in fields out of the saturation range. Figure 5(c) shows the MR data (solid circles) superimposed to the PMR signal (open circles).

\section{CONCLUSIONS}

In this paper, we present a novel characteristic of the perturbative magnetoresistance measurement technique, which was not explored by previous papers. We measured the 1 st harmonic of the resistance signal, modulated by a small AC field perpendicular to a DC field. We named the signal obtained by the PMR signal. Equations (2), (5), and (6) show that the PMR signal depends on the product of the second derivative of the magnetic energy with respect to the equilibrium position of the magnetization times the first derivative of the resistance with respect to the equilibrium position of the magnetization. A parameter sensitive to the 2nd derivative is, for instance, the switching field of the magnetization that is defined as the point of instability close to the saturation state. It is difficult to measure the switching or nucleation field in the low field regime by usual techniques (see Refs. 19 and 27 to understand the difference between switching and nucleation fields). We verified that the amplitude of the AC field cannot be larger than a threshold above which the PMR line shape changes significantly. The PMR technique is very effective to measure the magnetic phenomena that vary significantly in the low field regime, such as the magnetization switching. ${ }^{28-31}$ Certainly, the principle of functioning of PMR and the results presented in this paper add a novelty to the set of techniques that use the AMR phenomenon to probe the magnetization state in magnetic thin films and multilayers. ${ }^{32}$ Also, it will be of interest to scientists and engineers working with the development of magnetic instrumentation that operates in the low magnetic field regime.

\section{ACKNOWLEDGMENTS}

The authors gratefully acknowledge Axel Hoffmann (MSD, Argonne National Laboratory) for helpful comments and discussions on the results. The work has been support by Brazilian agencies, CNPq, CAPES, FINEP, and FAPERN.
${ }^{1}$ S. Tumanski, Handbook of Magnetic Measurements: Series in Sensors (CRC Press, Boca Raton, 2011).

${ }^{2}$ B. Bhushan, Springer Handbook of Nanotechnology (Springer, Heidelberg, 2004).

${ }^{3}$ S. Foner, Rev. Sci. Instrum. 30, 548 (1959).

${ }^{4}$ M. Alderighi, G. Bevilacqua, V. Biancalana, A. Khanbekyan, Y. Dancheva, and L. Moi, Rev. Sci. Instrum. 84, 125105 (2013).

${ }^{5}$ J. R. Kirtley, L. Paulius, A. J. Rosenberg, J. C. Palmstrom, C. M. Holland, E. M. Spanton, D. Schiessl, C. L. Jermain, J. Gibbons, Y.-K.-K. Fung, M. E. Huber, D. C. Ralph, M. B. Ketchen, G. W. Gibson, Jr., and K. A. Moler, Rev. Sci. Instrum. 87, 093702 (2016).

${ }^{6}$ T. Dumelow, M. M. Xavier, Jr., F. A. O. Cabral, and C. Chesman, J. Magn. Magn. Mater. 226-230, 2063 (2001).

${ }^{7}$ H. J. Richter, K. A. Hempel, and J. Pfeiffer, Rev. Sci. Instrum. 59, 1388 (1988).

${ }^{8}$ P. J. Flanders, Rev. Sci. Instrum. 61, 839 (1990).

${ }^{9}$ N. Shirato, H. Krishna, A. K. Gangopadhyay, and R. Kalyanaraman, Proc. SPIE 7767, 77670Q (2010)

${ }^{10}$ A. Stupakiewicz, A. Chizhik, M. Tekielak, A. Zhukov, J. Gonzalez, and A. Maziewski, Rev. Sci. Instrum. 85, 103702 (2014).

${ }^{11}$ L. Flajšman, M. Urbánek, V. Křižáková, M. Vaňatka, I. Turčan, and T. Šikola, Rev. Sci. Instrum. 87, 053704 (2016).

${ }^{12}$ V. K. Arkad'ev, J. Russ. Phys.-Chem. Soc. 44, 165 (1912).

${ }^{13}$ J. H. E. Griffiths, Nature 158, 670 (1946).

${ }^{14}$ C. Kittel, Phys. Rev. 73, 155 (1948).

${ }^{15}$ I. Harward, T. O'Keevan, A. Hutchison, V. Zagorodnii, and Z. Celinski, Rev. Sci. Instrum. 82, 095115 (2011).

${ }^{16}$ B. Hillebrands, Rev. Sci. Instrum. 70, 1589 (1999).

${ }^{17}$ T. R. McGuire and R. I. Potter, IEEE Trans. Magn. 11, 1018 (1975).

${ }^{18}$ M. N. Baibich, J. M. Broto, A. Fert, F. Nguyen Van Dau, F. Petroff, P. Etienne, G. Creuzet, A. Friederich, and J. Chazelas, Phys. Rev. Lett. 61, 2472 (1988).

${ }^{19}$ A. Aharoni, Introduction to the Theory of Ferromagnetism (Oxford University Press, Oxford, 2000).

${ }^{20}$ D. Venus, F. Hunte, I. N. Krivorotov, T. Gredig, and E. Dan Dahlberg, J. Appl. Phys. 93, 8609 (2003); D. Venus, F. Hunteb, and E. Dan Dahlbergb, J. Magn. Magn. Mater. 286, 191 (2005).

${ }^{21}$ R. D. Barnard, Physica B 217, 221 (1996).

${ }^{22}$ D. K. Schroder, Semiconductor Material and Device Characterization, 3rd ed. (Wiley, Hoboken, 2006).

${ }^{23}$ E. C. Stoner and E. P. Wohlfarth, IEEE Trans. Magn. 27, 3475 (1991).

${ }^{24}$ A. B. Oliveira, R. L. Rodriguez-Suarez, S. Michea, H. Veja, A. Azevedo, S. M. Rezende, C. Aliaga, and J. Denardin, J. Appl. Phys. 116, 033910 (2014).

${ }^{25}$ R. Alvarez, J. M. García-Martín, M. Macías-Montero, L. Gonzalez-Garcia, J. C. González, V. Rico, J. Perlich, J. Cotrino, A. R. González-Elipe, and A. Palmero, Nanotechnology 24, 045604 (2013).

${ }^{26}$ A. Aharoni, J. Appl. Phys. 82, 1281 (1997); A. B. Oliveira, S. M. Rezende, and A. Azevedo, Phys. Rev. B 78, 024423 (2008).

${ }^{27}$ A. Hubert and R. Schäfer, Magnetic Domains, the Analysis of Magnetic Microstructures (Springer, 2000).

${ }^{28}$ R. D. McMichael, M. D. Stiles, P. J. Chen, and W. F. Egelhoff, Jr., Phys. Rev. B 58, 8605 (1998).

${ }^{29}$ A. Harres and J. Geshev, J. Phys.: Condens. Matter 24, 326004 (2012).

${ }^{30}$ A. B. Oliveira, R. da Costa, A. Melo, and C. Chesman, "Perturbative magnetoresistance technique used to investigate FM/AF coupled bilayers," in March Meeting, Denver, Co, 2014.

${ }^{31}$ A. B. Oliveira, R. da Costa, A. Melo, and C. Chesman, "Static Magnetic Properties of Films Measured by Means of Angular Perturbative Magnetoresistance," in March Meeting, Baltimore, Md, 2016.

${ }^{32}$ E. Dan Dahlberg, K. Riggs, and G. A. Prinz, J. Appl. Phys. 63, 4270 (1988). 